PROCEEDINGS OF THE

AMERICAN MATHEMATICAL SOCIETY

Volume 129, Number 7 , Pages 1927-1937

S 0002-9939(00)05870-6

Article electronically published on November 22, 2000

\title{
THE RELATIVE PLURICANONICAL STABILITY FOR 3-FOLDS OF GENERAL TYPE
}

\author{
MENG CHEN
}

(Communicated by Ron Donagi)

\begin{abstract}
The aim of this paper is to improve a theorem of János Kollár by a different method. For a given smooth complex projective threefold $X$ of general type, suppose the plurigenus $P_{k}(X) \geq 2$. Kollár proved that the $(11 k+5)$ canonical map is birational. Here we show that either the $(7 k+3)$-canonical map or the $(7 k+5)$-canonical map is birational and that the $(13 k+6)$-canonical map is stably birational onto its image. Suppose $P_{k}(X) \geq 3$. Then the $m$ canonical map is birational for $m \geq 10 k+8$. In particular, $\phi_{12}$ is birational whenever $p_{g}(X) \geq 2$ and $\phi_{11}$ is birational whenever $p_{g}(X) \geq 3$.
\end{abstract}

\section{INTRODUCTION}

Let $X$ be a smooth projective 3 -fold of general type defined over $\mathbb{C}$ and denote by $\phi_{m}$ the $m$-canonical map of $X$, which is the rational map associated with the linear system $\left|m K_{X}\right|$. Let $P_{k}(X):=h^{0}\left(X, \mathcal{O}_{X}\left(k K_{X}\right)\right)$ for any positive integer $k$. We usually call $P_{k}(X)$ the $k$-th plurigenus of $X$ which is a birational invariant. For a given positive integer $m_{0}$, we say that $\phi_{m_{0}}$ is stably birational if $\phi_{m}$ is birational onto its image for all $m \geq m_{0}$. Since the Kodaira dimension $\operatorname{kod}(X)=3, \phi_{m}$ is birational for $m \gg 0$. In this paper, we consider the following

Problem. Suppose $P_{k}(X) \geq 2$. For which value $m_{0}(k)$, does $\left|m_{0}(k) K_{X}\right|$ define a stably birational map onto its image?

In 1986, Kollár ( 5 , Corollary 4.8]) first gave an effective result and proved that the $(11 k+5)$-canonical map is birational if $P_{k}(X) \geq 2$. However, his method cannot tell whether $\phi_{m}$ is still birational for all $m>11 k+5$. On the other hand, it seems to us that the number $11 k+5$ is not the optimal one. This paper aims to present a better result as the following

Main Theorem. Let $X$ be a nonsingular projective threefold of general type and suppose $P_{k}(X) \geq 2$. Then the following hold:

(i) either $\phi_{7 k+3}$ or $\phi_{7 k+5}$ is birational onto its image;

(ii) $\phi_{13 k+6}$ is stably birational onto its image;

(iii) $\phi_{10 k+8}$ is stably birational provided that $P_{k}(X) \geq 3$.

In particular, $\phi_{12}$ is stably birational if $p_{g}(X) \geq 2$ and $\phi_{11}$ is stably birational if $p_{g}(X) \geq 3$.

Received by the editors December 12, 1998 and, in revised form, November 12, 1999.

1991 Mathematics Subject Classification. Primary 14C20, 14E05, 14E35.

The author was supported in part by the National Natural Science Foundation of China. 
Noting that the main obstacle which prevents Kollár's method from getting a better bound is the case when $X$ admits a rational pencil of certain surfaces of general type, we shall mainly make a special study of this situation in an alternative way. First we build some birationality criteria for adjoint systems on a surface of general type. Then we reduce the problem to the surface case while finding suitable divisors on the threefold whose restrictions to the surface satisfy those criteria. The Kawamata-Viehweg vanishing theorem plays a key role throughout our argument.

Definition. Let $X$ be a normal projective variety and $D$ be a Weil divisor on $X$. Denote by $\Phi_{|D|}$ the natural rational map defined by the linear system $|D| .|D|$ is called base point free if it has neither fixed components nor base points.

If $|L|$ is a linear system on $X$ without fixed components and $h^{0}(X, L) \geq 2$, we mean a general irreducible element $S$ of $|L|$ as follows:

(1) If $\operatorname{dim} \Phi_{|L|}(X) \geq 2$, then $S$ is a general member of $|L|$.

(2) If $\operatorname{dim} \Phi_{|L|}(X)=1$, then $L$ is linearly equivalent to a union of distinct reduced irreducible divisors of the same type. Explicitly, $L \sim_{\operatorname{lin}} \sum S_{i}$. We mean by $S$ a general $S_{i}$.

$X$ is called minimal if the canonical divisor $K_{X}$ is nef, i.e. $K_{X} \cdot C \geq 0$ for all proper curves $C \subset X$.

$X$ is said to be of general type if the Kodaira dimension $\operatorname{kod}(X)=\operatorname{dim}(X)$.

$X$ is said to have only terminal singularities according to Reid ([7) if the following two conditions hold:

(i) for some integer $r \geq 1, r K_{X}$ is Cartier;

(ii) for some resolution $f: Y \longrightarrow X, K_{Y}=f^{*}\left(K_{X}\right)+\sum a_{i} E_{i}$ for $0<a_{i} \in \mathbb{Q}$ for all $i$, where the $E_{i}$ vary all the exceptional divisors on $Y$.

\section{Preparation}

Throughout our argument, the Kawamata-Viehweg vanishing theorem will be always employed as a much more effective tool. We shall use it in the following form.

Vanishing Theorem ([3] or [10]). Let $X$ be a nonsingular complete variety, $D \in$ $\operatorname{Div}(X) \otimes \mathbb{Q}$. Assume the following two conditions:

(1) $D$ is nef and big;

(2) the fractional part of $D$ has supports with only normal crossings.

Then $H^{i}\left(X, \mathcal{O}_{X}\left(\ulcorner D\urcorner+K_{X}\right)\right)=0$ for $i>0$, where $\ulcorner D\urcorner$ is the round-up of $D$, i.e. the minimum integral divisor with $\ulcorner D\urcorner-D \geq 0$.

Another important principle that is tacitly used throughout the text is due to Tankeev (9]). Explicitly, on a smooth projective variety $X$, if we have a base point free system $|M|$ and an effective divisor $D$, we want to study the birationality of the map $\Phi_{|D+M|}$. Now let $S$ be a general irreducible element of $|M|$. Then $S$ is a smooth divisor on $X$ by Bertini's theorem. Suppose we have known that $\Phi_{|D+M|}$ can distinguish general irreducible elements and that $\left.\Phi_{|D+M|}\right|_{S}$ is birational. Then Tankeev's principle implies the birationality of $\Phi_{|D+M|}$.

Lemma 1.1 ([8, Corollary 2]). Let $S$ be a nonsingular algebraic surface, $L$ be a nef divisor on $S, L^{2} \geq 10$ and let $\phi$ be a map defined by $\left|L+K_{S}\right|$. If $\phi$ is not birational, then $S$ contains a base point free pencil $E^{\prime}$ with $L \cdot E^{\prime}=1$ or $L \cdot E^{\prime}=2$. 
Lemma 1.2. Let $S$ be a nonsingular projective surface of general type and suppose $L$ is a divisor with $h^{0}(S, L) \geq 2$. Then $h^{0}\left(S, K_{S}+L\right) \geq 2$. In particular, if $\chi\left(\mathcal{O}_{S}\right) \geq$ 3 , then $h^{0}\left(S, K_{S}+L\right) \geq 4$.

Proof. Taking a general irreducible element $C$ in the moving part of $|L|$, then $C$ is a nef divisor, $C \leq L$ and $C$ is a curve of genus $\geq 2$. By R-R on the surface $S$, we have

$$
h^{0}\left(S, K_{S}+L\right) \geq h^{0}\left(S, K_{S}+C\right) \geq \frac{1}{2}\left(K_{S} \cdot C+C^{2}\right)+\chi\left(\mathcal{O}_{S}\right) .
$$

It is easy to get the result.

Lemma 1.3. Let $S$ be a nonsingular projective surface of general type, $L$ be a nef divisor, $L^{2} \geq 3$ and $\operatorname{dim} \Phi_{|L|}(S)=2$. Then $\left|K_{S}+2 L\right|$ gives a birational map.

Proof. We have $(2 L)^{2} \geq 12$. If $\Phi_{\left|K_{S}+2 L\right|}$ is not birational, then according to Lemma 1.1 , there is a base point free pencil $E^{\prime}$ such that $2 L \cdot E^{\prime} \leq 2$, i.e. $L \cdot E^{\prime}=1$. Since $\operatorname{dim} \Phi_{|L|}(S)=2$ and $E^{\prime}$ is a curve of genus $\geq 2$, we see that $L \cdot E^{\prime} \geq 2$, a contradiction.

Lemma 1.4. Let $S$ be a nonsingular projective surface of general type, $L_{i}$ be a divisor on $S$ such that $\operatorname{dim} \Phi_{\left|L_{i}\right|}(S) \geq i$ for $i=1$, 2 . Then $\left|K_{S}+2 L_{2}+L_{1}\right|$ gives a birational map.

Proof. Modulo blowing-ups, we can suppose that the $\left|L_{i}\right|$ is base point free for $i=1,2$. This means that $L_{2}$ is nef and big and that $L_{1}$ is nef.

If the system $\left|L_{2}\right|$ gives a birational map, then so does $\left|K_{S}+2 L_{2}+L_{1}\right|$, because $K_{S}+L_{1}$ is effective by Lemma 1.2 .

Otherwise, we have $L_{2}^{2} \geq 2$. Now we have $\left(2 L_{2}+L_{1}\right)^{2} \geq 12$. If $\left|K_{S}+2 L_{2}+L_{1}\right|$ does not give a birational map, then, by Lemma 1.1, there is a free pencil $E^{\prime}$ on $S$ such that

$$
\left(2 L_{2}+L_{1}\right) \cdot E^{\prime} \leq 2 .
$$

This means $L_{2} \cdot E^{\prime}=1$. Note that $E^{\prime}$ is a curve of genus $\geq 2$ and $\left|L_{2}\right|$ gives a generically finite map. The Riemann-Roch theorem on the curve $E^{\prime}$ tells that $\operatorname{deg}\left(\left.L_{2}\right|_{E^{\prime}}\right) \geq 2$. We have derived a contradiction.

Lemma 1.5. Let $X$ be a nonsingular projective 3-fold of general type. Suppose that $L_{i}$ is a divisor on $X$ such that $\operatorname{dim} \Phi_{\left|L_{i}\right|}(X) \geq i$ for $i=1,2,3$. Then

$$
\left|K_{X}+2 L_{3}+L_{2}+L_{1}\right|
$$

gives a birational map.

Proof. Take a birational modification $\pi: X^{\prime} \longrightarrow X$ according to Hironaka such that the $\left|\pi^{*}\left(L_{i}\right)\right|$ are all base point free for $i>0$. On $X^{\prime}$, we can study the system $\left|K_{X^{\prime}}+2 \pi^{*}\left(L_{3}\right)+\pi^{*}\left(L_{2}\right)+\pi^{*}\left(L_{1}\right)\right|$. Let $M_{i}$ be the moving part of $\left|\pi^{*}\left(L_{i}\right)\right|$. We have

$$
\left|K_{X^{\prime}}+2 M_{3}+M_{2}+M_{1}\right| \subset\left|K_{X^{\prime}}+2 \pi^{*}\left(L_{3}\right)+\pi^{*}\left(L_{2}\right)+\pi^{*}\left(L_{1}\right)\right| .
$$

Therefore, for simplicity, we can suppose from the beginning that the $\left|L_{i}\right|$ are base point free on $X$. So $L_{3}$ is nef and big under this assumption. 
Step 1. Verifying that $K_{X}+2 L_{3}+L_{2}$ is effective.

We have $\operatorname{dim} \Phi_{\left|L_{2}\right|}(X) \geq 2$. So a general member $S \in\left|L_{2}\right|$ is a nonsingular projective surface of general type. Using the vanishing theorem to the exact sequence

$$
0 \longrightarrow \mathcal{O}_{X}\left(K_{X}+2 L_{3}\right) \longrightarrow \mathcal{O}_{X}\left(K_{X}+2 L_{3}+S\right) \longrightarrow \mathcal{O}_{S}\left(K_{S}+\left.2 L_{3}\right|_{S}\right) \longrightarrow 0,
$$

we get the surjective map

$$
H^{0}\left(X, K_{X}+2 L_{3}+S\right) \longrightarrow H^{0}\left(S, K_{S}+\left.2 L_{3}\right|_{S}\right) \longrightarrow 0 .
$$

From Lemma 1.2, we know $K_{S}+\left.2 L_{3}\right|_{S}$ is effective, so is $K_{X}+2 L_{3}+L_{2}$.

Step 2. Reduction to surface case.

Taking a 1-dimensional sub-system of $\left|L_{1}\right|$, then this system defines a rational map onto $\mathbb{P}^{1}$. Taking further blowing-up if necessary, we can also suppose that this system defines a morphism $f: X \longrightarrow \mathbb{P}^{1}$. Taking the Stein factorization of $f$, one obtains a derived fibration $g: X \longrightarrow C$. A general fibre of $f$ can be written as a disjoint union $\sum F_{i}$. Let $F$ be a general fibre of $g$; then it is a nonsingular projective surface of general type and we have $F \leq L_{1}$. Now considering the system $\left|K_{X}+2 L_{3}+L_{2}+\sum F_{i}\right|$, it can distinguish general fibres of $g$ because $K_{X}+2 L_{3}+L_{2}$ is effective and $2 L_{3}+L_{2}$ is nef and big. Using the vanishing theorem again, we have

$$
\left.\left|K_{X}+2 L_{3}+L_{2}+\sum F_{i}\right|\right|_{F}=\left|K_{F}+2 L_{3}^{\prime}+L_{2}^{\prime}\right|,
$$

where $L_{3}^{\prime}:=\left.L_{3}\right|_{F}$ and $L_{2}^{\prime}:=\left.L_{2}\right|_{F}$. Lemma 1.4 tells that the right system gives a birational map, so does $\left|K_{X}+2 L_{3}+L_{2}+L_{1}\right|$. The proof is completed.

Lemma 1.6. Let $X$ be a nonsingular variety of dimension $n, D \in \operatorname{Div}(X) \otimes \mathbb{Q}$ be $a \mathbb{Q}$-divisor on $X$. Then we have the following:

(i) if $S$ is a smooth irreducible divisor on $X$ and $S$ is not a fractional component of $D$, then $\left.\ulcorner D\urcorner\right|_{S} \geq\left\ulcorner\left. D\right|_{S}\right\urcorner$;

(ii) if $\pi: X^{\prime} \longrightarrow X$ is a birational morphism, then $\pi^{*}(\ulcorner D\urcorner) \geq\left\ulcorner\pi^{*}(D)\right\urcorner$.

Proof. These are trivial.

Lemma 1.7. Let $X$ be a nonsingular projective threefold of general type. Let $D$ be a divisor on $X$ with $h^{0}(X, D) \geq 2$ and suppose $|D|$ has no fixed components. Denote by $F$ a general irreducible element of $|D|$. If $L$ is another divisor such that $\operatorname{dim} \Phi_{|L|}(F) \geq 1$, then $m K_{X}+L+D$ is effective and $\operatorname{dim} \Phi_{\left|m K_{X}+L+D\right|}(F) \geq 1$ for all $m \geq 2$.

Proof. According to the 3-dimensional MMP ([4] and [6]), $X$ has a minimal model $X_{0}$ which is normal projective with only $\mathbb{Q}$-factorial terminal singularities. Let $\alpha: X \rightarrow X_{0}$ be the contraction which is a rational map. Take a common resolution $X^{\prime}$ with $\pi^{\prime}: X^{\prime} \longrightarrow X$ and $\pi: X^{\prime} \longrightarrow X_{0}$ such that $\pi=\alpha \circ \pi^{\prime}$ and that

(1) both $\left|\pi^{\prime *}(L)\right|$ and $\left|\pi^{\prime *}(D)\right|$ have no base points (they may have fixed components);

(2) $\pi^{*}\left(K_{X_{0}}\right)$ has supports with only normal crossings.

This is possible because of Hironaka's big theorem. Since $\pi^{\prime *}\left(m K_{X}+L+D\right) \leq$ $m K_{X^{\prime}}+\pi^{\prime *}(L)+\pi^{\prime *}(D)$ and

$$
\begin{aligned}
\pi^{\prime}{ }_{*} \mathcal{O}_{X^{\prime}}\left(m K_{X^{\prime}}+\pi^{\prime *}(L)+\pi^{\prime *}(D)\right) & =\mathcal{O}_{X}\left(m K_{X}+L+D\right) \\
& =\pi^{\prime}{ }_{*} \pi^{\prime *} \mathcal{O}_{X}\left(m K_{X}+L+D\right),
\end{aligned}
$$


then $h^{0}\left(X^{\prime}, \pi^{\prime *}\left(m K_{X}+L+D\right)\right)=h^{0}\left(X^{\prime}, m K_{X^{\prime}}+\pi^{\prime *}(L)+\pi^{\prime *}(D)\right)$, so

$$
\Phi_{\left|\pi^{\prime *}\left(m K_{X}+L+D\right)\right|} \text { and } \Phi_{\left|m K_{X^{\prime}}+\pi^{\prime *}(L)+\pi^{\prime *}(D)\right|}
$$

have the same behavior. Let $S$ be a general irreducible element of the moving part of $\left|\pi^{\prime *}(D)\right|$; then $\operatorname{dim} \Phi_{\left|\pi^{\prime *}(L)\right|}(S) \geq 1$ by assumption. Therefore it is sufficient to show

$$
\operatorname{dim} \Phi_{\left|m K_{X^{\prime}}+\pi^{\prime *}(L)+\pi^{\prime *}(D)\right|}(S) \geq 1
$$

for $m \geq 2$. Let $H$ be the moving part of $\left|\pi^{\prime *}(L)\right|$; then $H$ is nef since $|H|$ is base point free. We have

$$
\left|K_{X^{\prime}}+\left\ulcorner(m-1) \pi^{*} K_{X_{0}}\right\urcorner+H+S\right| \subset\left|m K_{X^{\prime}}+\pi^{\prime *}(L)+\pi^{\prime *}(D)\right| .
$$

The Kawamata-Viehweg vanishing theorem gives

$$
\begin{aligned}
& \left.\left|K_{X^{\prime}}+\left\ulcorner(m-1) \pi^{*} K_{X_{0}}\right\urcorner+H+S\right|\right|_{S} \\
& =\left|K_{S}+\left\ulcorner(m-1) \pi^{*} K_{X_{0}}\right\urcorner\right|_{S}+M|\supset| K_{S}+\ulcorner B\urcorner+M \mid,
\end{aligned}
$$

where $B:=\left.(m-1) \pi^{*} K_{X_{0}}\right|_{S}$ is nef and big on $S$ and $M:=\left.H\right|_{S}$. From the assumption, we have $h^{0}(S, M) \geq 2$. Choosing a 1-dimensional sub-system $|C|$ in $|M|$, modulo blowing-ups, we can suppose $|C|$ to be base point free. Also from the vanishing theorem, we have

$$
\left.\left|K_{S}+\ulcorner B\urcorner+C\right|\right|_{C}=\left|K_{C}+D\right|,
$$

where $D:=\left.\ulcorner B\urcorner\right|_{C}$ is a divisor on the curve $C$ with positive degree since $D \geq\left\ulcorner\left. B\right|_{C}\right\urcorner$ by Lemma 1.6(i). Because $g(C) \geq 2$, we have $h^{0}\left(K_{C}+D\right) \geq 2$. This means $\left|K_{C}+D\right|$ gives a generically finite map and

$$
\operatorname{dim} \Phi_{\left|K_{S}+\ulcorner B\urcorner+C\right|}(C)=1 ;
$$

thus $K_{X^{\prime}}+\left\ulcorner(m-1) \pi^{*} K_{X_{0}}\right\urcorner+\pi^{\prime *}(L)+\pi^{\prime *}(D)$ is effective and the image of $S$ through the map defined by this divisor is at least 1 . The proof is completed.

\section{Proof of the main theorem}

2.1 Basic formula. Let $X$ be a nonsingular projective threefold, $f: X \longrightarrow C$ be a fibration onto a nonsingular curve $C$. From the spectral sequence:

$$
E_{2}^{p, q}:=H^{p}\left(C, R^{q} f_{*} \omega_{X}\right) \Rightarrow E^{n}:=H^{n}\left(X, \omega_{X}\right),
$$

we get by direct calculation that

$$
\begin{gathered}
h^{2}\left(X, \mathcal{O}_{X}\right)=h^{1}\left(C, f_{*} \omega_{X}\right)+h^{0}\left(C, R^{1} f_{*} \omega_{X}\right), \\
q(X):=h^{1}\left(X, \mathcal{O}_{X}\right)=b+h^{1}\left(C, R^{1} f_{*} \omega_{X}\right),
\end{gathered}
$$

where $b$ denotes the genus of $C$. 
2.2 Review of Kollár's technique. Let $X$ be a smooth projective 3-fold of general type and suppose $P_{k}(X) \geq 2$. Choose a 1-dimensional sub-system of $\left|k K_{X}\right|$ and replace $X$ by a birational model $X^{\prime}$ where this pencil defines a morphism $g: X^{\prime} \longrightarrow \mathbb{P}^{1}$. (For simplicity, we can suppose $X^{\prime}=X$.) Let $S$ be a general irreducible element of this pencil. Then a general fibre of $g$ is a disjoint union of some surfaces with the same type as $S$ and $S$ is a smooth projective surface of general type. Let $t=k(2 p+1)+p$. Then $H^{0}\left(\omega_{X}^{t}\right)=H^{0}\left(\mathbb{P}^{1}, g_{*} \omega_{X}^{t}\right)$ and we have an injection $\mathcal{O}(1) \hookrightarrow g_{*} \omega_{X}^{k}$, and hence an injection $\mathcal{O}(2 p+1) \hookrightarrow g_{*} \omega_{X}^{k(2 p+1)}$. This gives an injection

$$
\mathcal{O}(2 p+1) \otimes g_{*} \omega_{X}^{p} \hookrightarrow g_{*} \omega_{X}^{t},
$$

where $\mathcal{O}(2 p+1) \otimes g_{*} \omega_{X}^{p}=\mathcal{O}(1) \otimes g_{*} \omega_{X / \mathbb{P}^{1}}^{p}$. Now it is well-known that $g_{*} \omega_{X / \mathbb{P}^{1}}^{p}$ is a sum of line bundles of non-negative degree on $\mathbb{P}^{1}$. If $p \geq 5$, the local sections of $g_{*} \omega_{X}^{p}$ give a birational map for $S$ and all these extend to global sections of $\mathcal{O}(2 p+1) \otimes g_{*} \omega_{X}^{p}$. Moreover its sections separate the fibres from each other, and hence $\phi_{t}$ is a birational map for $X$.

From the above method, according to [1] and [1], we have

(1) $\phi_{5 k+2}$ is generically finite for $X$ if $S$ is not a surface with $p_{g}(S)=q(S)=0$ and $K_{S_{0}}^{2}=1$, where $S_{0}$ is the minimal model of $S$. Otherwise, we have at least $\operatorname{dim} \phi_{5 k+2}(X) \geq 2$;

(2) $\phi_{7 k+3}$ is birational for $X$ if $S$ is not a surface with

$$
\left(K_{S_{0}}^{2}, p_{g}(S)\right)=(1,2) \text { or }(2,3) .
$$

2.3 Proof of the main theorem. According to the 3-dimensional MMP, we can suppose $X$ to be a minimal model with at worst $\mathbb{Q}$-factorial terminal singularities. This means that $K_{X}$ is a nef and big $\mathbb{Q}$-divisor. We begin from a minimal model in order to make use of the Kawamata-Viehweg vanishing theorem.

Theorem 2.3.1. Let $X$ be a nonsingular projective 3-fold of general type and suppose $P_{k}(X) \geq 2$. Then either $\phi_{7 k+3}$ or $\phi_{7 k+5}$ is birational.

Proof. Suppose $X$ is a minimal model with at worst $\mathbb{Q}$-factorial terminal singularities. Choose a 1-dimensional sub-system $\Lambda$ of $\left|k K_{X}\right|$ and take a birational modification $\pi: X^{\prime} \longrightarrow X$ such that

(i) $X^{\prime}$ is nonsingular;

(ii) $\pi^{*} \Lambda$ gives a morphism;

(iii) the fractional part of $\pi^{*}\left(K_{X}\right)$ has supports with only normal crossings.

This is possible because of Hironaka's big theorem. Set $g_{1}:=\Phi_{\Lambda} \circ \pi$ and let $X^{\prime} \stackrel{f_{1}}{\longrightarrow} W_{1} \stackrel{s_{1}}{\longrightarrow} \mathbb{P}^{1}$ be the Stein factorization of $g_{1}$. Denote $b:=g\left(W_{1}\right)$, the geometric genus of the curve $W_{1}$.

If $b>0$, then the moving part of $\Lambda$ is base point free. Let $\sum S_{i}$ be the moving part of $\Lambda$; then $\sum S_{i} \leq k K_{X}$ and a general $S_{i}$ is a smooth projective surface of general type, since the singularities on $X$ are isolated. Using Kawamata's vanishing theorem $([4])$ to $\mathbb{Q}$-Cartier Weil divisors on minimal threefold $X$, we see that $\left|(a+1) K_{X}+\sum S_{i}\right|$ can distinguish general $S_{i}$ for $a>0$ and

$$
H^{0}\left(X,(a+1) K_{X}+\sum S_{i}\right) \longrightarrow \bigoplus H^{0}\left(S_{i},(a+1) K_{S_{i}}\right)
$$

is surjective. Therefore it is obvious that $\phi_{m}$ is effective whenever $m \geq k+2$, generically finite whenever $m \geq 2 k+2$, birational whenever $m \geq 2 k+4$. 
So, from now on, we can suppose that $b=0$. We have a fibration $f_{1}: X^{\prime} \longrightarrow \mathbb{P}^{1}$. Let $F$ be a general fibre of $f_{1}$. By virtue of 2.2(2), we can suppose that $F$ is a surface with invariants $\left(K_{F_{0}}^{2}, p_{g}(F)\right)=(1,2)$ or $(2,3)$, where $F_{0}$ is the minimal model of $F . F$ is the moving part of $\pi^{*} \Lambda$ and $F \leq_{\mathbb{Q}} \pi^{*}\left(k K_{X}\right)$. We automatically have $q(F)=0$. First we study the system $\left|K_{X^{\prime}}+\left\ulcorner k \pi^{*}\left(K_{X}\right)\right\urcorner+F\right|$. For a general fibre $F$, the vanishing theorem gives that

$$
\left.\left|K_{X^{\prime}}+\left\ulcorner k \pi^{*}\left(K_{X}\right)\right\urcorner+F\right|\right|_{F}=\left|K_{F}+\left\ulcorner k \pi^{*}\left(K_{X}\right)\right\urcorner\right|_{F} \mid,
$$

where $\left.\left\ulcorner k \pi^{*}\left(K_{X}\right)\right\urcorner\right|_{F}$ is effective. This means that $(2 k+1) K_{X^{\prime}}$ is effective and $\operatorname{dim} \phi_{2 k+1}(F) \geq 1$. By Lemma 1.7, we see that $m K_{X^{\prime}}$ is effective and $\operatorname{dim} \phi_{m}(F) \geq$ 1 for $m \geq 3 k+3$.

Actually, we have $\operatorname{dim} \phi_{3 k+2}(F)=2$. In fact, we have

$$
\left.\left|K_{X^{\prime}}+\left\ulcorner(2 k+1) \pi^{*}\left(K_{X}\right)\right\urcorner+F\right|\right|_{F} \supset\left|K_{F}+M_{2 k+1}\right|_{F} \mid,
$$

where $M_{2 k+1}$ is the moving part of $\left|\left\ulcorner(2 k+1) \pi^{*} K_{X}\right\urcorner\right|$. It is easy to check that $\left|K_{F}+M_{2 k+1}\right|_{F} \mid$ gives a generically finite map because $q(F)=0$ and $p_{g}(F)>0$. Thus

$$
\operatorname{dim} \Phi_{\left|K_{X^{\prime}}+\left\ulcorner(2 k+1) \pi^{*}\left(K_{X}\right)\right\urcorner+F\right|}(F) \geq 2 .
$$

We have $\left|K_{X^{\prime}}+\left\ulcorner 2(3 k+2) \pi^{*}\left(K_{X}\right)\right\urcorner+F\right| \subset\left|(7 k+5) K_{X^{\prime}}\right| . K_{X^{\prime}}+\left\ulcorner 2(3 k+2) \pi^{*}\left(K_{X}\right)\right\urcorner$ is effective by the above argument. So $\left|K_{X^{\prime}}+\left\ulcorner 2(3 k+2) \pi^{*}\left(K_{X}\right)\right\urcorner+F\right|$ can distinguish general fibre $F$. On the other hand, the Kawamata-Viehweg vanishing theorem gives

$$
\begin{aligned}
\left.\left|K_{X^{\prime}}+\left\ulcorner 2(3 k+2) \pi^{*}\left(K_{X}\right)\right\urcorner+F\right|\right|_{F} & =\left|K_{F}+\left\ulcorner 2(3 k+2) \pi^{*}\left(K_{X}\right)\right\urcorner\right|_{F} \mid \\
& \supset\left|K_{F}+2 L_{3 k+2}\right|,
\end{aligned}
$$

where $L_{3 k+2}:=\left.M_{3 k+2}\right|_{F}$. It is sufficient to show that $\left|K_{F}+2 L_{3 k+2}\right|$ gives a birational map for $F$. We have already known that $\left|L_{3 k+2}\right|$ gives a generically finite map for $F$. Excluding the fixed components of $\left|L_{3 k+2}\right|$, we can suppose that $\left|L_{3 k+2}\right|$ are moving on the surface $F$. So $L_{3 k+2}$ is nef. If $\left|L_{3 k+2}\right|$ gives a birational map, then so does $\left|K_{F}+2 L_{3 k+2}\right|$. Otherwise,

$$
L_{3 k+2}^{2} \geq 2\left(h^{0}\left(F, L_{3 k+2}\right)-2\right) .
$$

Consider the following three natural maps:

$$
\begin{aligned}
& H^{0}\left(X^{\prime}, M_{3 k+2}\right) \stackrel{\alpha}{\longrightarrow} H^{0}\left(F, L_{3 k+2}\right), \\
& H^{0}\left(X^{\prime}, K_{X^{\prime}}+\left\ulcorner(2 k+1) \pi^{*}\left(K_{X}\right)\right\urcorner+F\right) \\
& \qquad \stackrel{\beta}{\longrightarrow} H^{0}\left(F, K_{F}+\left.\left\ulcorner(2 k+1) \pi^{*}\left(K_{X}\right)\right\urcorner\right|_{F}\right) \longrightarrow 0, \\
& H^{0}\left(X^{\prime},(3 k+2) K_{X^{\prime}}\right) \stackrel{\gamma}{\longrightarrow} H^{0}\left(F,(3 k+2) K_{F}\right)
\end{aligned}
$$

where $\beta$ is surjective by the Kawamata-Viehweg vanishing theorem. We see that

$$
\operatorname{dim}_{\mathbb{C}}(\operatorname{im}(\alpha))=\operatorname{dim}_{\mathbb{C}}(\operatorname{im}(\gamma)) \geq \operatorname{dim}_{\mathbb{C}}(\operatorname{im}(\beta))=h^{0}\left(F, K_{F}+D_{2 k+1}\right)
$$

where $D_{2 k+1}:=\left.\left\ulcorner(2 k+1) \pi^{*}\left(K_{X}\right)\right\urcorner\right|_{F}$ and $h^{0}\left(F, D_{2 k+1}\right) \geq 2$. So

$$
h^{0}\left(F, K_{F}+D_{2 k+1}\right) \geq 4
$$

according to Lemma 1.2, because we have $\chi\left(\mathcal{O}_{F}\right) \geq 3$ in this case. Thus

$$
L_{3 k+2}^{2} \geq 2\left(h^{0}\left(F, L_{3 k+2}\right)-2\right) \geq 2\left(\operatorname{dim}_{\mathbb{C}}(\operatorname{im}(\alpha))-2\right) \geq 4
$$


and then $\left|K_{F}+2 L_{3 k+2}\right|$ gives a birational map by Lemma 1.3. So $\phi_{7 k+5}$ is birational.

Finally, for all $m \geq 10 k+7$, set $t:=m-7 k-5 \geq 3 k+2$; then $\operatorname{dim} \phi_{t}(F) \geq 1$. In particular, $t K_{X^{\prime}}$ is effective. So $\phi_{m}$ is birational for all $m \geq 10 k+7$ in this case.

Corollary 2.3.1. Let $X$ be an irregular nonsingular 3-fold of general type and suppose $P_{k}(X) \geq 2$. Then $\phi_{7 k+3}$ is birational. Therefore at least $\phi_{143}$ is birational according to Kollár and Fletcher.

Proof. In the proof of the last theorem, if $b>0$, then $\phi_{m}$ is birational for $m \geq 2 k+4$. If $b=0$, we can use the formula of $q(X)$ to the fibration $f_{1}: X^{\prime} \longrightarrow \mathbb{P}^{1}$. When $q(X)>0$, then we must have $q(F)>0$. Then $\Phi_{\left|3 K_{F}\right|}$ is birational for the fibre $F$, and so is $\Phi_{\left|(7 k+3) K_{X}\right|}$ by $2.2(2)$. Moreover, we have $P_{20}(X) \geq 2$ for any irregular 3 -fold of general type according to Kollár ([5]) and Fletcher ([2]). Thus $\phi_{143}$ is birational.

Theorem 2.3.2. Let $X$ be a nonsingular projective threefold of general type and suppose $P_{k}(X) \geq 2$. Then $\phi_{m}$ is birational for $m \geq 13 k+6$.

Proof. Suppose $X$ is a minimal model with at worst $\mathbb{Q}$-factorial terminal singularities. Make a birational modification $\pi: X^{\prime} \longrightarrow X$ such that:

(i) $X^{\prime}$ is nonsingular;

(ii) $\left|k K_{X^{\prime}}\right|$ gives a morphism;

(iii) the fractional part of $\pi^{*}\left(K_{X}\right)$ has supports with only normal crossings.

Set $g:=\Phi_{\left|k K_{X}\right|} \circ \pi$ and $W^{\prime}:=\overline{\Phi_{\left|k K_{X}\right|}(X)}$. Let $X^{\prime} \stackrel{f}{\longrightarrow} W \stackrel{s}{\longrightarrow} W^{\prime}$ be the Stein factorization of $g$.

We would like to formulate our proof through two steps as follows.

Case 1. $\operatorname{dim} \phi_{k}(X) \geq 2$.

Set $k K_{X^{\prime}} \sim_{\text {lin }} M_{k}+Z_{k}$, where $M_{k}$ is the moving part and $Z_{k}$ is the fixed part. Then a general member $S \in\left|M_{k}\right|$ is an irreducible nonsingular projective surface of general type. Write $K_{X^{\prime}}=\pi^{*}\left(K_{X}\right)+\sum a_{i} E_{i}$, where the $E_{i}$ are exceptional divisors for $\pi, 0<a_{i} \in \mathbb{Q}$ for each $i$. Obviously, $\left\ulcorner\pi^{*}\left(K_{X}\right)\right\urcorner \leq K_{X^{\prime}}$. Because $h^{0}\left(X^{\prime},\left\ulcorner\pi^{*}\left(k K_{X}\right)\right\urcorner\right)=h^{0}\left(X^{\prime}, k K_{X^{\prime}}\right)$, we can see that $M_{k}$ is actually also the moving part of $\left|\left\ulcorner\pi^{*}\left(k K_{X}\right)\right\urcorner\right|$. Thus we have

$$
\pi^{*}\left(k K_{X}\right) \geq_{\mathbb{Q}} M_{k}+\sum b_{i} E_{i},
$$

where $0 \leq b_{i} \in \mathbb{Q}$ for each $i$.

We claim that $m K_{X^{\prime}}$ is always effective for $m \geq 2 k+1$. In fact, for any $t \in \mathbb{Z}^{+}$, we consider the system

$$
\left|K_{X^{\prime}}+\left\ulcorner\pi^{*}\left((t+k) K_{X}\right)\right\urcorner+S\right| .
$$

It is a sub-system of $\left|(2 k+t+1) K_{X^{\prime}}\right|$. By the Kawamata-Viehweg vanishing theorem, we have a surjective map

$$
H^{0}\left(X^{\prime}, K_{X^{\prime}}+\left\ulcorner\pi^{*}\left((t+k) K_{X}\right)\right\urcorner+S\right) \longrightarrow H^{0}\left(S, K_{S}+\left.\left\ulcorner\pi^{*}\left((t+k) K_{X}\right)\right\urcorner\right|_{S}\right) \longrightarrow 0 .
$$

Noting that $\left\ulcorner\pi^{*}\left((t+k) K_{X}\right)\right\urcorner \geq\left\ulcorner\pi^{*}\left(t K_{X}\right)\right\urcorner+M_{k}$, also by Lemma 1.6(i), it is sufficient to show that $K_{S}+\left\ulcorner\left.\pi^{*}\left(t K_{X}\right)\right|_{S}\right\urcorner+\left.M_{k}\right|_{S}$ is effective. When $t=0$, then $h^{0}\left(S, K_{S}+\left.M_{k}\right|_{S}\right) \geq 2$ by Lemma 1.2 , because $h^{0}\left(S,\left.M_{k}\right|_{S}\right) \geq 2$. When $t>0$, choose a 1-dimensional sub-system $|C|$ in the moving part of $\left|M_{k}\right|_{S} \mid$. Modulo blowing-ups, we can suppose $|C|$ to be free from base points and then $C$ is nef and 
$C \leq\left. M_{k}\right|_{S}$. We have $g(C) \geq 2$. Because $\left.\pi^{*}\left(t K_{X}\right)\right|_{S}$ is a nef and big $\mathbb{Q}$-divisor on $S$, by the Kawamata-Viehweg vanishing theorem, we also get a surjective map

$$
H^{0}\left(S, K_{S}+\left\ulcorner\left.\pi^{*}\left(t K_{X}\right)\right|_{S}\right\urcorner+C\right) \longrightarrow H^{0}\left(C, K_{C}+D\right) \longrightarrow 0,
$$

where $D:=\left.\left\ulcorner\left.\pi^{*}\left(t K_{X}\right)\right|_{S}\right\urcorner\right|_{C}$ is a divisor on $C$ with positive degree. Thus we have $h^{0}\left(C, K_{C}+D\right) \geq 2$. This leads to the effectiveness of $(2 k+t+1) K_{X^{\prime}}$. Moreover, actually we have proved that $\operatorname{dim} \phi_{m}(S) \geq 1$ for $m \geq 2 k+1$.

Now we prove that $\phi_{3 k+1}$ is generically finite. Considering the system

$$
\left|K_{X^{\prime}}+\left\ulcorner 2 k \pi^{*}\left(K_{X}\right)\right\urcorner+M_{k}\right|,
$$

as we have shown above that $(2 k+1) K_{X^{\prime}}$ is effective, so $\left|K_{X^{\prime}}+\left\ulcorner 2 k \pi^{*}\left(K_{X}\right)\right\urcorner+M_{k}\right|$ can distinguish general $S$. By the Kawamata-Viehweg vanishing theorem, we have

$$
\left.\left|K_{X^{\prime}}+\left\ulcorner 2 k \pi^{*}\left(K_{X}\right)\right\urcorner+S\right|\right|_{S}=\left|K_{S}+\left\ulcorner 2 k \pi^{*}\left(K_{X}\right)\right\urcorner\right|_{S} \mid .
$$

We have

$$
\left|K_{S}+\left\ulcorner 2 k \pi^{*}\left(K_{X}\right)\right\urcorner\right|_{S}|\supset| K_{S}+\left\ulcorner\left. k \pi^{*}\left(K_{X}\right)\right|_{S}\right\urcorner+\left.M_{k}\right|_{S} \mid .
$$

Noting that $h^{0}\left(S,\left.M_{k}\right|_{S}\right) \geq 2, K_{S}+\left\ulcorner\left. k \pi^{*}\left(K_{X}\right)\right|_{S}\right\urcorner \geq K_{S}+\left.M_{k}\right|_{S}$, which is also effective by Lemma 1.2 , and $\left.k \pi^{*}\left(K_{X}\right)\right|_{S}$ is a nef and big $\mathbb{Q}$-divisor on $S$, it is easy to verify that $\left|K_{S}+\left\ulcorner\left. k \pi^{*}\left(K_{X}\right)\right|_{S}\right\urcorner+M_{k}\right|_{S} \mid$ gives a generically finite map. In fact, choose a 1-dimensional sub-system $|C|$ in the moving part of $\left|M_{k}\right|_{S} \mid$. For the same reason, we can suppose $|C|$ to be free from base points. $\left|K_{S}+\left\ulcorner\left. k \pi^{*}\left(K_{X}\right)\right|_{S}\right\urcorner+C\right|$ can distinguish general $C$, and we have

$$
\left.\left|K_{S}+\left\ulcorner\left. k \pi^{*}\left(K_{X}\right)\right|_{S}\right\urcorner+C\right|\right|_{C}=\left|K_{C}+D\right|,
$$

where $D$ is a divisor on $C$ with positive degree. Because $g(C) \geq 2$, it follows that $h^{0}\left(K_{C}+D\right) \geq 2$ and $\left|K_{C}+D\right|$ gives a generically finite map.

Finally, we want to show that $\phi_{m}$ is birational for $m \geq 9 k+4$. Let $t:=m-7 k-3$; then $t \geq 2 k+1$. Denote by $M_{3 k+1}$ the moving part of $\left|(3 k+1) K_{X^{\prime}}\right|$ and by $M_{t}$ the moving part of $\left|t K_{X^{\prime}}\right|$. We have

$$
\left|K_{X^{\prime}}+\left\ulcorner(t+6 k+2) \pi^{*}\left(K_{X}\right)\right\urcorner+M_{k}\right| \subset\left|m K_{X^{\prime}}\right| .
$$

Because $t+6 k+3>2 k+1, K_{X^{\prime}}+\left\ulcorner(t+6 k+2) \pi^{*}\left(K_{X}\right)\right\urcorner$ is effective; thus the left system in the above can distinguish general $S$. Furthermore, the vanishing theorem gives

$$
\left.\left|K_{X^{\prime}}+\left\ulcorner(t+6 k+2) \pi^{*}\left(K_{X}\right)\right\urcorner+M_{k}\right|\right|_{S}=\left|K_{S}+L\right|,
$$

where $L:=\left.\left\ulcorner(t+6 k+2) \pi^{*}\left(K_{X}\right)\right\urcorner\right|_{S} \geq\left. 2 M_{3 k+1}\right|_{S}+\left.M_{t}\right|_{S}$. By Lemma 1.4, $\left|K_{S}+L\right|$ gives a birational map, and so does $\left|m K_{X^{\prime}}\right|$.

Case 2. $\operatorname{dim} \phi_{k}(X)=1$.

In this case, $W$ is a nonsingular curve of genus $b$. Let $F$ be a general fibre of $f$; then $F$ is an irreducible smooth projective surface of general type. We have $M_{k} \sim_{\operatorname{lin}} \sum F_{i}$, where the $F_{i}$ are fibres of $f$ for each $i$.

By a parallel argument as in the proof of Theorem 2.3.1, we see that $\phi_{m}$ is birational for $m \geq 2 k+4$ if $b>0$. And if $b=0$ while $F$ is a surface with the invariants $\left(K_{F_{0}}^{2}, p_{g}(F)\right)=(1,2)$ or $(2,3)$, then $\phi_{m}$ is birational for $m \geq 10 k+7$.

Otherwise, we use Kollár's method. From 2.2, we know that $\phi_{7 k+3}$ is birational and $\operatorname{dim} \phi_{5 k+2}(X) \geq 2$. Thus, by Lemma $1.7, m K_{X^{\prime}}$ is effective for $m \geq 6 k+$ 4. Since we have $\left.\left|K_{X^{\prime}}+\left\ulcorner(5 k+2) \pi^{*}\left(K_{X}\right)\right\urcorner+F\right|\right|_{F}=\left|K_{F}+D\right|$ where $D:=$ $\left.\left\ulcorner(5 k+2) \pi^{*}\left(K_{X}\right)\right\urcorner\right|_{F}$ is effective and $h^{0}(F, D) \geq 2$, we see that $K_{F}+D$ is effective 
and thus $(6 k+3) K_{X^{\prime}}$ is effective. So $\phi_{m}$ is birational for $m \geq 13 k+6$, which means that $\phi_{13 k+6}$ is stably birational.

Theorem 2.3.3. Let $X$ be a nonsingular projective threefold of general type and suppose $P_{k}(X) \geq 3$. Then $\phi_{m}$ is birational for all $m \geq 10 k+8$.

Proof. When $\operatorname{dim} \phi_{k}(X) \geq 2$, we know from Case 1 of Theorem 2.3.2 that $\phi_{m}$ is birational for $m \geq 9 k+4$. When $\left|k K_{X}\right|$ is composed of a pencil, from the proof of Theorem 2.3.1, we see that $\phi_{k}$ will derive a fibration $f: X^{\prime} \longrightarrow W$ onto a nonsingular curve. If $b:=g(W)>0$, then $\phi_{m}$ is birational for $m \geq 2 k+4$.

The remaining case is the one when $b=0$. We have an injection $\mathcal{O}(2) \hookrightarrow f_{*} \omega_{X^{\prime}}^{k}$. So, for each $p>0$, we have

$$
\mathcal{O}(1) \otimes f_{*} \omega_{X^{\prime} / \mathbb{P}^{1}}^{p}=\mathcal{O}(2 p+1) \otimes f_{*} \omega_{X^{\prime}}^{p} \hookrightarrow f_{*} \omega_{X^{\prime}}^{k(p+1)+p} .
$$

Thus Kollár's method tells that $\phi_{6 k+5}$ is birational, $\phi_{4 k+3}$ is generically finite and that $\operatorname{dim} \phi_{3 k+2}(X) \geq 2$. Now using our method, we can see that $m K_{X^{\prime}}$ is effective for $m \geq 4 k+4$ by Lemma 1.7. Since $(4 k+3) K_{X^{\prime}}$ is also effective, $\phi_{m}$ is birational for $m \geq 10 k+8$.

Corollary 2.3.2. Let $X$ be a nonsingular projective threefold of general type and suppose $p_{g}(X) \geq 3$. Then $\phi_{m}$ is birational for $m \geq 11$.

Proof. Keep the same notation as in the proof of Theorem 2.3.2. When $\operatorname{dim} \phi_{1}(X)$ $\geq 2$, we set $L_{3}:=4 K_{X^{\prime}}, L_{2}=L_{1}:=K_{X^{\prime}}$. Then $\left|L_{3}\right|$ gives a generically finite map by virtue of Case 1, Theorem 2.3.2. Using Lemma 1.5, we see that $\mid K_{X^{\prime}}+2 L_{3}+$ $L_{2}+L_{1} \mid$ gives a birational map. Thus $\phi_{11}$ is birational.

When $\operatorname{dim} \phi_{1}(X)=1$, we see from the proof of Theorem 2.3.3 that $\phi_{11}$ is also birational.

Theorem 2.3.1, Theorem 2.3.2, Theorem 2.3.3 and Corollary 2.3.2 imply the main theorem.

\section{Open problems}

3.1. Let $X$ be a nonsingular projective variety of general type of dimension $n$. We define

$k_{0}(X):=\min \left\{k \mid P_{k}(X) \geq 2\right\} ;$

$k_{s}(X):=\min \left\{k \mid \phi_{m}\right.$ is birational for $\left.m \geq k\right\}$, which is called the canonical stability of $X$;

$\mu_{s}(X):=\frac{k_{s}(X)}{k_{0}(X)}$, which is called the relative canonical stability of $X$. Obviously, $\mu_{s}(X)$ is a birational invariant.

$\mu_{s}(n):=\sup \left\{\mu_{s}(X) \mid X\right.$ is a $n$-fold of general type $\}$, which is called the $n$-th relative canonical stability.

It is well-known that $\mu_{s}(1)=3$ and $\mu_{s}(2)=5([1])$. From the main theorem, we have $\mu_{s}(3) \leq 16$. What is the exact value of $\mu_{s}(3)$ ? It is also interesting to study $\mu_{s}(n)$ for $n \geq 4$, even if we don't know whether we should have $\mu_{s}(n)<+\infty$.

3.2. We would like to ask a very natural question which never happens in the surface case.

Question. Do there exist a smooth projective threefold $X$ of general type and two positive integers $k_{1}<k_{2}$ such that $\phi_{k_{1}}$ is birational while $\phi_{k_{2}}$ is not birational? 
Of course, it may happen for some threefold that $P_{k_{1}}>P_{k_{2}}$ even if $k_{1}<k_{2}$. But we have not found any counterexample yet to the above question.

\section{ACKNOWLEDGMENT}

I would like to thank the Abdus Salam International Centre for Theoretical Physics, Trieste, Italy for support during my visit. I also wish to thank Prof. L. Ein and Prof. M. S. Narasimhan for encouragement. Thanks are also due to Prof. J. Kollár and Prof. M. Reid, who privately gave me much more useful information on the problem considered in this paper. During the preparation of this note, I visited both the Università di Roma II and the Università degli Studi di Padova by the invitation of Prof. Ciro Ciliberto and Prof. Ezio Stagnaro whom I take this chance to thank for their hospitality. Finally, the referee's skillful comments greatly helped me to organize this paper into the present form.

\section{REFERENCES}

[1] W. Barth, C. Peter, A. Van de Ven, Compact Complex Surface, Springer-Verlag 1984. MR 86c:32026

[2] A. R. Fletcher, Contributions to Riemann-Roch in projective 3-folds with only canonical singularities and application, Proc. Sympos. Pure Math. 46, Amer. Math. Soc. Providence, 1987, 221-232. MR 89h:14032

[3] Y. Kawamata, A generalization of Kodaira-Ramanujam's vanishing theorem, Math. Ann. 261(1982), 43-46. MR 84i:14022

[4] Y. Kawamata, K. Matsuda, K. Matsuki, Introduction to the minimal model problem, Adv. Stud. Pure Math. 10 (1987), 283-360. MR 89e:14015

[5] J. Kollár, Higher direct images of dualizing sheaves I, Ann. of Math. 123(1986), 11-42. MR 87c:14038

[6] J. Kollár, S. Mori, Birational geometry of algebraic varieties, Cambridge Univ. Press, 1998. MR 2000b: 14018

[7] M. Reid, Canonical 3-folds, Journées de Géométrie Algébrique d'Angers, A. Beauville (editor), Sijthoff and Noordhoff, Alphen aan den Rijn, 1980, pp. 273-310. MR 82i:14025

[8] I. Reider, Vector bundles of rank 2 and linear systems on algebraic surfaces, Ann. of Math. 127(1988), 309-316. MR 89e:14038

[9] S. G. Tankeev, On n-dimensional canonically polarized varieties and varieties of fundamental type, Izv. A. N. SSSR, Sér. Math. 35(1971), 31-44. MR 43:3261

[10] E. Viehweg, Vanishing theorems, J. reine angew. Math. 335(1982), 1-8. MR 83m:14011

[11] G. Xiao, Finitude de l'application bicanonique des surfaces de type général, Bull. Soc. Math. France 113(1985), 23-51. MR 87a:14035

Department of Applied Mathematics, Tongui University, Shanghai, 200092, People's Republic of China

E-mail address: mchen@mail.tongji.edu.cn 